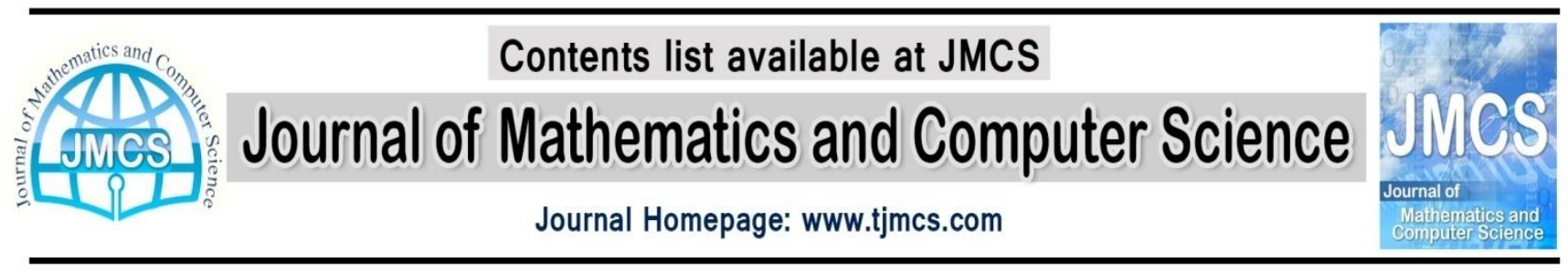

\title{
Lattice Valued Fuzzy Soft Grills
}

\author{
Vildan Çetkin 1,", Halis Aygün ${ }^{1,+}$ \\ ${ }^{1}$ Kocaeli University, Department of Mathematics, Umuttepe Campus, 41380, Kocaeli-TURKEY \\ vcetkin@gmail.com \\ +halis@kocaeli.edu.tr
}

Article history:

Received 05 Jan 2015

Accepted 23 Jan 2015

Available online 30 January 2015

\begin{abstract}
The present paper is devoted to the study of fuzzy soft grill structure. The notions of fuzzy soft grill and fuzzy soft grill base are defined and the connections between them are given. Two types of second order image and reimage of fuzzy soft grill base is defined and also some of their properties are observed.
\end{abstract} Keywords: fuzzy soft set, fuzzy soft grill, grill base.

\section{Introduction}

In 1999, Molodtsov [16] proposed a completely new concept called soft set theory to model uncertainty, which associates a set with a set of parameters. Later, Maji et al [14] introduced the concept of fuzzy soft set which combines fuzzy sets and soft sets. Soft set and fuzzy soft set theories have a rich potential for applications in several directions. So far, many scholars working in diverse areas have handled the soft set and fuzzy soft set theories in different aspects (see $[4,5,11,12,13,15,18])$. Also, Aygünoğlu et al.[7] studied the topological structure of fuzzy soft sets based on the sense of Sostak [19].

The convergence theory of grills provide a good tool for interpreting topological structures, and plays an important role in topology. In general topology, the notion of grill was first proposed by Choquet [10] in 1947, which has been observed as an excellent tool for studying different topological concepts. In fuzzy setting, the concept of fuzzy grills on fuzzy topological spaces was initiated by Azad [2], basically for the study of proximities in fuzzy spaces. Subsequently, Srivastava and Gupta [20] and Chattopadhyay et al.[9] investigated fuzzy basic proximity by use of fuzzy grills. Recently, some researchers extended these studies to the broader framework of fuzzy topology. In [8], the authors have studied fuzzy compactness, fuzzy almost compactness etc. via fuzzy grills.

In this paper, we first introduced the notion of fuzzy soft grill and fuzzy soft grill base based on a complete lattice. We gave the relations between fuzzy soft grill and fuzzy soft grill base. Later we considered the second order preimages and the images of fuzzy soft grills and also investigated some of their properties. 


\section{Preliminaries}

Throughout this study, $X$ refers to an initial universe, $L$ and $M$ denote the complete lattice and the completely distributive lattice with the least elements $0_{L}, 0_{M}$ and the greatest elements $1_{L}, 1_{M}$, respectively and there is an order reversing involution ' on $L$.

Definition 2.1 [18] $f$ is called an $L$-fuzzy soft set on $X$, where $f$ is a mapping from $E$ into $L^{X}$, i.e., $f_{e} \triangleq f(e)$ is an $L$-fuzzy set on $X$, for each $e \in E$. The family of all $L$-fuzzy soft sets on $X$ is denoted by $\left(L^{X}\right)^{E}$.

Definition 2.2 $[17,18]$ Let $f$ and $g$ be two $L$-fuzzy soft set on $X$, then

(1) we say that $f$ is an $L$-fuzzy soft subset of $g$ and write $f \sqsubseteq g$ if $f_{e} \leq g_{e}$, for each $e \in E$.f and $g$ are called equal if $f$ 드 $g$ and $g$ $f$.

(2) the union of $f$ and $g$ is an $L$-fuzzy soft set $h=f \sqcup g$, where $h_{e}=f_{e} \vee g_{e}$, for each $e \in E$.

(3) the intersection of $f$ and $g$ on $X$ is an $L$-fuzzy soft set $h=f \sqcap g$, where $h_{e}=f_{e} \wedge g_{e}$, for each $e \in$ E.

(4) the complement of an $L$-fuzzy soft set $f$ is denoted by $f^{\prime}$, where $f^{\prime}: E \rightarrow L^{X}$ is a mapping given by $f_{e}^{\prime}=\left(f_{e}\right)^{\prime}$, for each $e \in E$.

\section{Definition 2.3 [18]}

(1) An $L$-fuzzy soft set $f$ on $X$ is called a null $L$-fuzzy soft set and denoted by $\Phi$, if $f_{e}(x)=0$, for each $e \in E, x \in X$.

(2) An $L$-fuzzy soft set $f$ on $X$ is called an absolute $L$-fuzzy soft set and denoted by $\widetilde{E}$, if $f_{e}(x)=1$, for each $e \in E, x \in X$. Clearly, $(\tilde{E})^{\prime}=\Phi, \phi^{\prime}=\tilde{E}$.

Proposition 2.1 [3] Let $\Delta$ be an index set and $f, g, f_{i}, g_{i} \in\left(L^{X}\right)^{E}$, for all $i \in \Delta$, then we have the following properties:

(1) $f \sqcap\left(\sqcup_{i \in \Delta} g_{i}\right)=\sqcup_{i \in \Delta}\left(f \sqcap g_{i}\right)$ and $f \sqcup\left(\Pi_{i \in \Delta} g_{i}\right)=\Pi_{i \in \Delta}\left(f \sqcup g_{i}\right)$.

(2) $\left(\Pi_{i \in \Delta} f_{i}\right)^{\prime}=\sqcup_{i \in \Delta} f_{i}{ }^{\prime}$ and $\left(\sqcup_{i \in \Delta} f_{i}\right)^{\prime}=\Pi_{i \in \Delta} f_{i}{ }^{\prime}$.

Definition 2.4 [6] Let $\varphi: X_{1} \rightarrow X_{2}$ and $\psi: E_{1} \rightarrow E_{2}$ be two functions, where $E_{1}$ and $E_{2}$ are parameter sets for the crisp sets $X_{1}$ and $X_{2}$, respectively. Then the pair $\varphi_{\psi}$ is called an $L$-fuzzy soft mapping from $X_{1}$ to $X_{2}$.

Definition 2.5 [6] Let $f$ and $g$ be two $L$-fuzzy soft set on $X_{1}$ and $X_{2}$, respectively and let $\varphi_{\psi}$ be an soft mapping from $X_{1}$ to $X_{2}$.

(1) The image of $f$ under the soft mapping $\varphi_{\psi}$, denoted by $\varphi_{\psi}(f)$, is the an $L$-fuzzy soft set on $X_{2}$ defined by for all $k \in E_{2}, y \in X_{2}$,

$\varphi_{\psi}(f)_{k}(y)=\left\{\begin{array}{lr}\bigvee_{y=\varphi(x)} \bigvee_{k=\psi(a)} f_{a}(x), & \text { if } x \in \varphi^{-1}(y) \\ 0, & \text { otherwise }\end{array}\right.$

(2) The preimage of $g$ under the soft mapping $\varphi_{\psi}$, denoted by $\varphi_{\psi}^{-1}(g)$, is an $L$-fuzzy soft set on $X_{1}$ denoted by 
$\varphi_{\psi}^{-1}(g)_{e}(x)=g_{\psi(e)}(\varphi(x))$, for all $e \in E_{1}, x \in X_{1}$.

If $\varphi$ and $\varphi$ are injective (surjective), then $\varphi_{\psi}$ is said to be injective (surjective).

(3) Let $\varphi_{\psi}$ be an $L$-fuzzy soft mapping from $X_{1}$ to $X_{2}$ and let $\varphi^{*}{ }_{\psi *}$ be an $L$-fuzzy soft mapping from $X_{2}$ to $X_{3}$. Then the composition of these mappings from $X_{1}$ to $X_{3}$ is defined as follows: $\varphi_{\psi} \circ \varphi *_{\psi *}=(\varphi \circ \varphi *)_{\psi \circ \psi *}$, where $\psi: E_{1} \rightarrow E_{2}$ and $\varphi *: E_{2} \rightarrow E_{3}$.

Proposition 2.2 [13] Let $X_{1}$ and $X_{2}$ be two universes, $f_{i} \in\left(L^{X_{1}}\right)^{E_{1}}$ and $g_{i} \in\left(L^{X_{2}}\right)^{E_{2}}$ for all $i \in \Delta$, where $\Delta$ is an index set. Then we have the following:

(1) $\varphi_{\psi}\left(\sqcup_{i \in \Delta} f_{i}\right)=\sqcup_{i \in \Delta} \varphi_{\psi}\left(f_{i}\right)$.

(2) $\varphi_{\psi}\left(\Pi_{i \in \Delta} f_{i}\right) \subseteq \Pi_{i \in \Delta} \varphi_{\psi}\left(f_{i}\right)$, the equality holds if $\varphi_{\psi}$ is injective.

(3) $\varphi_{\psi}^{-1}\left(\sqcup_{i \in \Delta} g_{i}\right)=\sqcup_{i \in \Delta} \varphi_{\psi}^{-1}\left(g_{i}\right)$ and $\varphi_{\psi}^{-1}\left(\Pi_{i \in \Delta} g_{i}\right)=\Pi_{i \in \Delta} \varphi_{\psi}^{-1}\left(g_{i}\right)$.

Throughout this study, let $E$ and $K$ be arbitrary nonempty sets viewed on the sets of parameters and $L, M$ be the complete lattices, unless otherwise stated.

\section{Fuzzy soft grill structure}

Definition 3.1 A mapping $\mathcal{G}: K \rightarrow M^{\left(L^{X}\right)^{E}}$ (where $\mathcal{G}_{k}:=\mathcal{G}(k):\left(L^{X}\right)^{E} \rightarrow M$ is a mapping for each $k \in$ $K)$ is called an $(L, M)$-fuzzy $(E, K)$-soft grill on $X$ if it satisfies the following conditions for each $k \in$ $K$ :

(SG1) $\mathcal{G}_{k}(\Phi)=0_{M}$ and $\mathcal{G}_{k}(\tilde{E})=1_{M}$.

(SG2) $\mathcal{G}_{k}(f \sqcup g) \leq \mathcal{G}_{k}(f) \vee \mathcal{G}_{k}(g)$, for each $f, g \in\left(L^{X}\right)^{E}$.

(SG3) If $f \subseteq g$, then $\mathcal{G}_{k}(f) \leq \mathcal{G}_{k}(g)$.

The pair $(X, \mathcal{G})$ is called an $(L, M)$-fuzzy $(E, K)$-soft grill space.

Let $\mathcal{G}^{1}$ and $\mathcal{G}^{2}$ be two $(L, M)$-fuzzy $(E, K)$-soft grills on $X$. We say $\mathcal{G}^{1}$ is finer than $\mathcal{G}^{2}$ denoted by $\left(\mathcal{G}^{1} \geq \mathcal{G}^{2}\right)$ or $\mathcal{G}^{2}$ is coarser than $\mathcal{G}^{1}$ if $\mathcal{G}_{k}^{1}(f) \leq \mathcal{G}_{k}^{2}(f)$ for each $k \in K$ and $f \in\left(L^{X}\right)^{E}$.

Notation 3.1 Let $\mathcal{B}: K \rightarrow M^{\left(L^{X}\right)^{E}}$ be a mapping, $k \in K$ and $f \in\left(L^{X}\right)^{E}$. We define the mapping $\langle\mathcal{B}\rangle: K \rightarrow M^{\left(L^{X}\right)^{E}}$ as follows:

$$
\langle\mathcal{B}\rangle_{k}(f)=\bigwedge\left\{\mathcal{B}_{k}(g) \mid f \sqsubseteq g\right\}
$$

Definition 3.2 A mapping $\mathcal{B}: K \rightarrow M^{\left({ }^{(}\right)^{E}}$ is called an $(L, M)$-fuzzy $(E, K)$-soft grill base on $X$, if it satisfies the following conditions for each $k \in K$ :

(GB1) $\mathcal{B}_{k}(\Phi)=0_{M}$ and $\mathcal{B}_{k}(\tilde{E})=1_{M}$.

$(\mathrm{GB} 2)\langle\mathcal{B}\rangle_{k}(f \sqcup g) \leq \mathcal{B}_{k}(f) \vee \mathcal{B}_{k}(g)$, for each $f, g \in\left(L^{X}\right)^{E}$.

Let $\mathcal{B}^{1}$ and $\mathcal{B}^{2}$ be $(L, M)$-fuzzy $(E, K)$-soft grill bases on $X$. We say $\mathcal{B}^{1}$ is finer than $\mathcal{B}^{2}$ if $\left\langle\mathcal{B}^{1}\right\rangle_{k}(f) \leq\left\langle\mathcal{B}^{2}\right\rangle_{k}(f)$ for each $k \in K$ and $f \in\left(L^{X}\right)^{E}$.

Remark 3.1 (1) If $\mathcal{G}$ is an $(L, M)$-fuzzy $(E, K)$-soft grill on $X$, then $\mathcal{G}$ is an $(L, M)$-fuzzy $(E, K)$-soft grill base on the same set with $\langle\mathcal{G}\rangle=\mathcal{G}$. 
(2) If a map $\mathcal{B}: K \rightarrow M^{\left(L^{X}\right)^{E}}$ is an $(L, M)$-fuzzy $(E, K)$-soft grill base, then by (GB2), $f \sqcup g=\tilde{E}$ implies $\mathcal{B}_{k}(f) \vee \mathcal{B}_{k}(g)=1_{M}$ for each $k \in K$.

Proposition 3.1 If $\mathcal{B}: K \rightarrow M^{\left(L^{X}\right)^{E}}$ is an $(L, M)$-fuzzy $(E, K)$-soft grill base, then $\langle\mathcal{B}\rangle$ is the coarsest $(L, M)$-fuzzy $(E, K)$-soft grill satisfying $\mathcal{B} \geq\langle\mathcal{B}\rangle$.

Proof. The conditions (SG1) and (SG3) are easily checked. For each $f \sqsubseteq f_{1}$ and $g \sqsubseteq g_{1}$, since $f \sqcup$ $g \sqsubseteq f_{1} \sqcup g_{1}$

$$
\langle\mathcal{B}\rangle_{k}(f \sqcup g) \leq\langle\mathcal{B}\rangle_{k}\left(f_{1} \sqcup g_{1}\right) \leq\langle\mathcal{B}\rangle_{k}\left(f_{1}\right) \vee\langle\mathcal{B}\rangle_{k}\left(g_{1}\right)
$$

for each $k \in K$. Since $M$ is completely distributive, then it can be easily seen that $\langle\mathcal{B}\rangle_{k}(f \sqcup g) \leq \mathcal{B}_{k}(f) \vee \mathcal{B}_{k}(g)$, for each $k \in K$.

Hence $\mathcal{B}$ is an $(L, M)$-fuzzy $(E, K)$-soft grill on $X$. Suppose that $\mathcal{G}$ is an $(L, M)$-fuzzy $(E, K)$-soft grill on $X$ satisfying $\mathcal{B} \geq \mathcal{G}$. So we have

$$
\langle\mathcal{B}\rangle_{k}(f)=\bigwedge\left\{\mathcal{B}_{k}(g) \mid f \sqsubseteq g\right\} \geq \bigwedge\left\{\mathcal{G}_{k}(g) \mid f \sqsubseteq g\right\}=\mathcal{G}_{k}(f)
$$

Theorem 3.1 If $\mathcal{H}: K \rightarrow M^{\left(L^{X}\right)^{E}}$ is a map satisfying the following condition:

(C) $\mathcal{H}_{k}(\Phi)=0_{M}$, for each $k \in K$, and for every finite index set $J$, if $\sqcup_{i \in J} g_{i}=\Phi$, then $\mathrm{\vee}_{i \in J} \mathcal{H}_{k}\left(g_{i}\right)=1_{M}$.

Let $\mathcal{B}^{\mathcal{H}}: K \rightarrow M^{\left(L^{X}\right)^{E}}$ be a mapping defined as follows:

$\mathcal{B}_{k}^{\mathcal{H}}(f)=\Lambda\left\{\vee_{i \in J} \mathcal{H}_{k}\left(g_{i}\right) \mid f=\sqcup_{i \in J} g_{i}\right\}$,

where the infimum is taken over for every finite index set $J$ such that $f=\sqcup_{i \in J} g_{i}$. Then the following properties are satisfied:

(1) $\mathcal{B}^{\mathcal{H}}$ is an $(L, M)$-fuzzy $(E, K)$-soft grill base on $X$.

(2) If $\mathcal{H} \geq \mathcal{B}$ and $\mathcal{B}$ is an $(L, M)$-fuzzy $(E, K)$-soft grill base on $X$, then $\left\langle\mathcal{B}^{\mathcal{H}}\right\rangle \geq\langle\mathcal{B}\rangle$.

Proof. (1) (GB1) By the condition $(\mathrm{C}), \mathcal{B}_{k}^{\mathcal{H}}(\widetilde{E})=1_{M}$ and $\mathcal{B}_{k}^{\mathcal{H}}(\Phi)=0_{M}$.

(GB2) For each two finite index sets $I$ and $J$ with $f_{1}=\sqcup_{i \in I} g_{i}$ and $f_{2}=\sqcup_{j \in J} h_{j}$, since $f_{1} \sqcup f_{2}=\left(\sqcup_{i \in I} g_{i}\right) \sqcup\left(\sqcup_{j \in J} h_{j}\right)$, by the definition of $\mathcal{B}^{\mathcal{H}}$, we have for each $k \in K$,

$$
\left\langle\mathcal{B}^{\mathcal{H}}\right\rangle_{k}\left(f_{1} \sqcup f_{2}\right) \leq\left(\vee_{i \in I} \mathcal{H}_{k}\left(g_{i}\right)\right) \vee\left(\vee_{j \in J} \mathcal{H}_{k}\left(g_{j}\right)\right)
$$

Since $M$ is completely distributive, if we take infimum over the families $\left\{f_{1}=\sqcup_{i \in I} g_{i}\right\}$ and $\left\{f_{2}=\sqcup_{j \in J} h_{j}\right\}$, we obtain

$\left\langle\mathcal{B}^{\mathcal{H}}\right\rangle_{k}\left(f_{1} \sqcup f_{2}\right) \leq \mathcal{B}_{k}^{\mathcal{H}}\left(f_{1}\right) \vee \mathcal{B}_{k}^{\mathcal{H}}\left(f_{2}\right)$, for all $f_{1}, f_{2} \in\left(L^{X}\right)^{E}$.

Then, $\mathcal{B}^{\mathcal{H}}$ is an $(L, M)$-fuzzy $(E, K)$-soft grill base on $X$.

(2) For each finite family $\left\{g_{i} \mid f \sqsubseteq \sqcup_{i \in I} g_{i}\right\}$, we have for each $k \in K$,

$$
\langle\mathcal{B}\rangle_{k}(f) \leq\langle\mathcal{B}\rangle_{k}\left(\sqcup_{i \in I} g_{i}\right) \leq \mathrm{v}_{i \in I} \mathcal{B}_{k}\left(g_{i}\right) \leq \mathrm{v}_{i \in I} \mathcal{H}_{k}\left(g_{i}\right)
$$

Thus, the desired inequality $\left\langle\mathcal{B}^{\mathcal{H}}\right\rangle \geq\langle\mathcal{B}\rangle$ is obtained.

Definition 3.3 Let $\left(X_{1}, \mathcal{G}^{1}\right)$ and $\left(X_{2}, \mathcal{G}^{2}\right)$ be an $(L, M)$-fuzzy $\left(E_{1}, K_{1}\right)$-soft grill space and $(L, M)$-fuzzy $\left(E_{2}, K_{2}\right)$-soft grill space, respectively. Then the function $\varphi_{\psi, \eta}:\left(X_{1}, \mathcal{G}^{1}\right) \rightarrow\left(X_{2}, \mathcal{G}^{2}\right)$ is said to be: 
(1) a grill map iff $\mathcal{G}_{\eta(k)}^{2}(g) \geq \mathcal{G}_{k}^{1}\left(\varphi_{\psi}^{-1}(g)\right)$, for each $k \in K_{1}, g \in\left(L^{X_{2}}\right)^{E_{2}}$.

(2) a grill preserving map iff $\mathcal{G}_{k}^{1}(f) \geq \mathcal{G}_{\eta(k)}^{2}\left(\varphi_{\psi}(f)\right)$, for each $k \in K_{1}, f \in\left(L^{X_{1}}\right)^{E_{1}}$.

Naturally the composition of grill maps (and also the grill preserving maps) is a grill map (and also a grill preserving map).

Proposition 3.2 Let $\mathcal{B}^{1}$ and $\mathcal{B}^{2}$ be an $(L, M)$-fuzzy $\left(E_{1}, K_{1}\right)$-soft grill base on $X_{1}$ and an $(L, M)$-fuzzy $\left(E_{2}, K_{2}\right)$-soft grill base on $X_{2}$, respectively. Then the following properties are satisfied:

(1) $\varphi_{\psi, \eta}:\left(X_{1},\left\langle\mathcal{B}^{1}\right\rangle\right) \rightarrow\left(X_{2},\left\langle\mathcal{B}^{2}\right\rangle\right)$ is a grill map iff $\mathcal{B}_{\eta(k)}^{2}(g) \geq\left\langle\mathcal{B}^{1}\right\rangle_{k}\left(\varphi_{\psi}^{-1}(g)\right)$, for each $k \in K_{1}$, $g \in\left(L^{X_{2}}\right)^{E_{2}}$.

(2) $\varphi_{\psi, \eta}:\left(X_{1},\left\langle\mathcal{B}^{1}\right\rangle\right) \rightarrow\left(X_{2},\left\langle\mathcal{B}^{2}\right\rangle\right)$ is a grill preserving map iff $\mathcal{B}_{k}^{1}(f) \geq\left\langle\mathcal{B}^{2}\right\rangle_{\eta(k)}\left(\varphi_{\psi}(f)\right)$, for each $k \in K_{1}, f \in\left(L^{X_{1}}\right)^{E_{1}}$.

(3) If $\mathcal{B}_{\eta(k)}^{2}(g) \geq \mathcal{B}^{1}{ }_{k}\left(\varphi_{\psi}^{-1}(g)\right)$, for each $k \in K_{1}, g \in\left(L^{X_{2}}\right)^{E_{2}}$, then $\varphi_{\psi, \eta}:\left(X_{1},\left\langle\mathcal{B}^{1}\right\rangle\right) \rightarrow\left(X_{2},\left\langle\mathcal{B}^{2}\right\rangle\right)$ is a grill map.

(4) If $\mathcal{B}_{k}^{1}(f) \geq \mathcal{B}^{2}{ }_{\eta(k)}\left(\varphi_{\psi}(f)\right)$, for each $k \in K_{1}, f \in\left(L^{X_{1}}\right)^{E_{1}}$, then $\varphi_{\psi, \eta}:\left(X_{1},\left\langle\mathcal{B}^{1}\right\rangle\right) \rightarrow\left(X_{2},\left\langle\mathcal{B}^{2}\right\rangle\right)$ is a grill preserving map.

Proof. Straightforward and therefore omitted.

\section{Second order image and preimage operators}

In this section, we consider the second order preimages and images of fuzzy soft grill bases.

Let the mapping $U: K_{1} \rightarrow M^{\left(L^{X_{1}}\right)^{E_{1}}}$ and $\mathcal{V}: K_{2} \rightarrow M^{\left(L^{X_{2}}\right)^{E_{2}}}$ be given.

The basic scheme for the second order image operator as follows:

(1) Image operator of the image operator:

$\left(\varphi_{\psi, \eta}\right)_{1}^{\Rightarrow}(\mathcal{U})_{k} *(g)=\mathrm{V}\left\{\mathrm{V}\left\{\mathcal{U}_{k}(f) \mid g=\varphi_{\psi}(f)\right\} \mid k^{*}=\eta(k)\right\}$, for each $k^{*} \in K_{2}, g \in\left(L^{X_{2}}\right)^{E_{2}}$

(2) Preimage operator of the image operator:

$\left(\varphi_{\psi, \eta}\right)_{2}^{\Rightarrow}(\mathcal{U})_{k} *(g)=\vee\left\{\mathcal{U}_{k}\left(\varphi_{\psi}^{-1}(g)\right) \mid k^{*}=\eta(k)\right\}$, for each $k^{*} \in K_{2}, g \in\left(L^{X_{2}}\right)^{E_{2}}$.

The basic scheme for the second order preimage operator as follows:

(1) Image operator of the preimage operator:

$\left(\varphi_{\psi, \eta}\right)_{1}^{\in}(\mathcal{V})_{k}(f)=\vee\left\{\mathcal{V}_{\eta(k)}(g) \mid f=\varphi_{\psi}^{-1}(g)\right\}$, for each $k \in K_{1}, f \in\left(L^{X_{1}}\right)^{E_{1}}$.

(2) Preimage operator of the preimage operator:

$\left(\varphi_{\psi, \eta}\right)_{2}^{\Leftarrow}(\mathcal{V})_{k}(f)=\mathcal{V}_{\eta(k)}\left(\varphi_{\psi}(f)\right)$, for each $k \in K_{1}, f \in\left(L^{X_{1}}\right)^{E_{1}}$.

Theorem 4.1 Let $\mathcal{B}$ be an $(L, M)$-fuzzy $\left(E_{2}, K_{2}\right)$-soft grill base on $X_{2}$. Then we have the following properties:

(1) If $\varphi_{\psi}^{-1}(h)=\Phi$ implies $\mathcal{B}_{k^{*}}(h)=0_{M}$, for each $k^{*} \in K_{2}$, then $\left(\varphi_{\psi, \eta}\right)_{1}^{\Leftarrow}(\mathcal{B})$ is an $(L, M)$-fuzzy $\left(E_{1}, K_{1}\right)$-soft grill base on $X_{1}$ and $\left\langle\left(\varphi_{\psi, \eta}\right)_{1}^{\rightleftarrows}(\mathcal{B})\right\rangle$ is the coarsest $(L, M)$-fuzzy $\left(E_{1}, K_{1}\right)$-soft grill for which $\varphi_{\psi, \eta}:\left(X_{1},\left\langle\left(\varphi_{\psi, \eta}\right)_{1}^{\models}(\mathcal{B})\right\rangle\right) \rightarrow\left(X_{2},\langle\mathcal{B}\rangle\right)$ is a grill map. 
(2) If $\varphi$ and $\psi$ are surjective, then $\left(\varphi_{\psi, \eta}\right)_{1}^{\Leftarrow}(\mathcal{B})$ is an $(L, M)$-fuzzy $\left(E_{1}, K_{1}\right)$-soft grill base on $X_{1}$.

Proof. (1) (GB1) Since $\varphi_{\psi}^{-1}\left(\tilde{E}_{2}\right)=\tilde{E}_{1},\left(\varphi_{\psi, \eta}\right)_{1}^{\models}(\mathcal{B})_{k}\left(\tilde{E}_{1}\right)=1_{M}$, for each $k \in K_{1}$. By the assumption $\left(\varphi_{\psi, \eta}\right)_{1}^{\Leftarrow}(\mathcal{B})_{k}\left(\Phi_{1}\right)=0_{M}$

(GB2) Suppose there exist $f_{1}, f_{2} \in\left(L^{X_{1}}\right)^{E_{1}}$ and $k \in K_{1}$ such that

$$
\left\langle\left(\varphi_{\psi, \eta}\right)_{1}^{\Leftarrow}(\mathcal{B})\right\rangle_{k}\left(f_{1} \sqcup f_{2}\right) \nsubseteq\left(\varphi_{\psi, \eta}\right)_{1}^{\Leftarrow}(\mathcal{B})_{k}\left(f_{1}\right) \vee\left(\varphi_{\psi, \eta}\right)_{1}^{\Leftarrow}(\mathcal{B})_{k}\left(f_{2}\right)
$$

By the definition of $\left(\varphi_{\psi, \eta}\right)_{1}^{\Leftarrow}(\mathcal{B})_{k}\left(f_{i}\right)$, for $i \in\{1,2\}$, there exist $h_{i} \in\left(L^{X_{2}}\right)^{E_{2}}$ with $f_{i}=\varphi_{\psi}^{-1}\left(h_{i}\right)$ such that

$$
\left\langle\left(\varphi_{\psi, \eta}\right)_{1}^{\Leftarrow}(\mathcal{B})\right\rangle_{k}\left(f_{1} \sqcup f_{2}\right) \not \mathcal{B}_{\eta(k)}\left(h_{1}\right) \vee \mathcal{B}_{\eta(k)}\left(h_{2}\right) .
$$

Since $\mathcal{B}$ is an $(L, M)$-fuzzy $\left(E_{2}, K_{2}\right)$-soft grill base, i.e.,

$\langle\mathcal{B}\rangle_{k^{*}}\left(h_{1} \sqcup h_{2}\right) \leq \mathcal{B}_{k^{*}}\left(h_{1}\right) \vee \mathcal{B}_{k^{*}}\left(h_{2}\right)$, for each $k^{*} \in K_{2}$, we have

$$
\left\langle\left(\varphi_{\psi, \eta}\right)_{1}^{\models}(\mathcal{B})\right\rangle_{k}\left(f_{1} \sqcup f_{2}\right) \$\langle\mathcal{B}\rangle_{\eta(k)}\left(h_{1} \sqcup h_{2}\right) .
$$

By the definition of $\langle B\rangle$, there exists $h \in\left(L^{X_{2}}\right)^{E_{2}}$ with $h_{1} \sqcup h_{2} \sqsubseteq h$ such that

$$
\left\langle\left(\varphi_{\psi, \eta}\right)_{1}^{\Leftarrow}(\mathcal{B})\right\rangle_{k}\left(f_{1} \sqcup f_{2}\right) \$ \mathcal{B}_{\eta(k)}(h) .
$$

On the other hand, $f_{1} \sqcup f_{2}=\varphi_{\psi}^{-1}\left(h_{1} \sqcup h_{2}\right) \sqsubseteq \varphi_{\psi}^{-1}(h)$. So,

$$
\left\langle\left(\varphi_{\psi, \eta}\right)_{1}^{\Leftarrow}(\mathcal{B})\right\rangle_{k}\left(f_{1} \sqcup f_{2}\right) \leq \mathcal{B}_{\eta(k)}(h) .
$$

It is a contradiction. Hence $\left(\varphi_{\psi, \eta}\right)_{1}^{\in}(\mathcal{B})$ is an $(L, M)$-fuzzy $\left(E_{1}, K_{1}\right)$-soft grill base on $X_{1}$.

Let $\varphi_{\psi, \eta}:\left(X_{1}, \mathcal{G}\right) \rightarrow\left(X_{2},\langle\mathcal{B}\rangle\right)$ be a grill map. For each $f \in\left(L^{X_{1}}\right)^{E_{1}}$ and $k \in K_{1}$, we have

$\left\langle\left(\varphi_{\psi, \eta}\right)_{1}^{\Leftarrow}(\mathcal{B})\right\rangle_{k}(f)=\wedge\left\{\mathcal{B}_{\eta(k)}(g) \mid f \sqsubseteq \varphi_{\psi}^{-1}(g)\right\} \geq \Lambda\left\{\mathcal{G}_{k}\left(\varphi_{\psi}^{-1}(g)\right) \mid f \sqsubseteq \varphi_{\psi}^{-1}(g)\right\} \geq \mathcal{G}_{k}(f)$.

(2) Since $\varphi, \psi$ are surjective, $\varphi_{\psi}^{-1}(h)=\Phi$ implies $h=\Phi$. So, $\mathcal{B}_{k^{*}}(\Phi)=0_{M}$ for each $k^{*} \in K_{2}$. By condition (1), it is straightforward.

Theorem 4.2 Let $\mathcal{B}$ be an $(L, M)$-fuzzy $\left(E_{2}, K_{2}\right)$-soft grill on $X_{2}$. If $\varphi_{\psi}^{-1}(h)=\Phi$ implies $\mathcal{B}_{k^{*}}(h)=$ $0_{M}$, for each $k^{*} \in K_{2}$ and also $\varphi, \psi$ are bijective functions, then $\left(\varphi_{\psi, \eta}\right)_{1}^{\Leftarrow}(\mathcal{B})$ is an $(L, M)$-fuzzy $\left(E_{1}, K_{1}\right)$-soft grill on $X_{1}$.

Proof. (SG1) and (SG2) are obvious.

(SG3) Let $f \sqsubseteq g$, for $f, g \in\left(L^{X_{1}}\right)^{E_{1}}$. Since $\varphi$ and $\psi$ are surjective, then there exists $h \in\left(L^{X_{2}}\right)^{E_{2}}$ with $\varphi_{\psi}^{-1}(h)=f$ and $g=f \sqcup g=\varphi_{\psi}^{-1}(h) \sqcup \varphi_{\psi}^{-1}\left(\varphi_{\psi}(g)\right)=\varphi_{\psi}^{-1}\left(h \sqcup \varphi_{\psi}(g)\right)$. This implies

$$
\left(\varphi_{\psi, \eta}\right)_{1}^{\Leftarrow}(\mathcal{B})_{k}(g) \geq \mathcal{B}_{\eta(k)}\left(h \sqcup \varphi_{\psi}(g)\right) \geq \mathcal{B}_{\eta(k)}(h) .
$$

Hence, $\left(\varphi_{\psi, \eta}\right)_{1}^{\Leftarrow}(\mathcal{B})_{k}(g) \geq\left(\varphi_{\psi, \eta}\right)_{1}^{\Leftarrow}(\mathcal{B})_{k}(f)$.

Theorem 4.3 Let $\varphi_{i}: X \rightarrow X_{i}, \psi_{i}: E \rightarrow E_{i}$ and $\eta_{i}: K \rightarrow K_{i}$ be functions for all $i \in \Gamma$. Let $\left\{\mathcal{B}_{i}\right\}_{i \in \Gamma}$ be a family of $(L, M)$-fuzzy $\left(E_{i}, K_{i}\right)$-soft grill bases on $X_{i}$ satisfying the following condition:

(C) For every finite subset $I$ of $\Gamma$, if $\sqcup_{i \in I}\left(\varphi_{\psi}\right)_{i}^{-1}\left(h_{i}\right)=\widetilde{E}_{X}$, then $\vee_{i \in I} \mathcal{B}^{i} k^{*}\left(h_{i}\right)=1_{M}$, for each $k^{*} \in$ $K_{i}$. We define a map 


$$
\begin{aligned}
\mathcal{B}:=\cup_{i \in \Gamma}\left(\left(\varphi_{\psi, \eta}\right)_{i}\right)_{1}^{\models}\left(\mathcal{B}^{i}\right): K & \rightarrow M^{\left(L^{X}\right)^{E}} \text { as } \\
\mathcal{B}_{k}(f) & =\Lambda\left\{\vee_{i \in I} \mathcal{B}_{\eta_{i}(k)}^{i}\left(h_{i}\right) \mid f=\sqcup_{i \in I}\left(\varphi_{\psi}\right)_{i}^{-1}\left(h_{i}\right)\right\},
\end{aligned}
$$

where the infimum is taken for every finite subset $I$ of $\Gamma$ such that $f=\sqcup_{i \in I}\left(\varphi_{\psi}\right)_{i}^{-1}\left(h_{i}\right)$. Then the following properties are satisfied:

(1) $\mathcal{B}$ is an $(L, M)$-fuzzy $(E, K)$-soft grill base on $X$ and $\langle\mathcal{B}\rangle$ is the coarsest $(L, M)$-fuzzy $(E, K)$-soft grill for which $\left(\varphi_{\psi, \eta}\right)_{i}:(X,\langle\mathcal{B}\rangle) \rightarrow\left(X_{i},\left\langle\mathcal{B}^{i}\right\rangle\right)$ is a grill map for all $i \in \Gamma$.

(2) A map $\varphi_{\psi, \eta}:\left(Y, \mathcal{G}^{\prime}\right) \rightarrow(X,\langle\mathcal{B}\rangle)$ is a grill map iff for each $i \in \Gamma,\left(\varphi_{\psi, \eta}\right)_{i} \circ \varphi_{\psi, \eta}:\left(Y, \mathcal{G}^{\prime}\right) \rightarrow$ $\left(X_{i},\left\langle\mathcal{B}^{i}\right\rangle\right)$ is a grill map.

(3) $\langle\mathcal{B}\rangle=\left\langle\mathrm{U}_{i \in \Gamma}\left(\left(\left(\varphi_{\psi, \eta}\right)_{i}\right)_{1}^{\Leftarrow}\right)\left(\left\langle\mathcal{B}^{i}\right\rangle\right)\right\rangle$.

Proof. (1) (GB1) By condition (C) and $\left(\varphi_{\psi}\right)_{i}^{-1}\left(\widetilde{E}_{X_{i}}\right)=\widetilde{E_{X}}, \mathcal{B}_{k}\left(\widetilde{E}_{X}\right)=1_{M}$ and $\mathcal{B}_{k}\left(\Phi_{X}\right)=0_{M}$, for each $k \in K$.

(GB2) Suppose there exist $f_{1}, f_{2} \in\left(L^{X}\right)^{E}$ such that $\langle\mathcal{B}\rangle_{k}\left(f_{1} \sqcup f_{2}\right) \nless \mathcal{B}_{k}\left(f_{1}\right) \vee \mathcal{B}_{k}\left(f_{2}\right)$. By the definition of $\mathcal{B}_{k}\left(f_{1}\right)$, there exists a finite subset $I$ of $\Gamma$ with

$$
f_{1}=\sqcup_{i \in I}\left(\varphi_{\psi}\right)_{i}^{-1}\left(h_{i}\right)
$$

such that

$$
\langle\mathcal{B}\rangle_{k}\left(f_{1} \sqcup f_{2}\right) \$\left(\vee_{i \in I} \mathcal{B}_{\eta_{i}(k)}^{i}\left(h_{i}\right)\right) \vee \mathcal{B}_{k}\left(f_{2}\right) .
$$

Again by the definition of $\mathcal{B}_{k}\left(f_{2}\right)$, there exists a finite subset $J$ of $\Gamma$ with

$$
f_{2}=\sqcup_{j \in J}\left(\varphi_{\psi}\right)_{j}^{-1}\left(g_{j}\right)
$$

such that

$$
\langle\mathcal{B}\rangle_{k}\left(f_{1} \sqcup f_{2}\right) \nsubseteq\left(\mathrm{v}_{i \in I} \mathcal{B}_{\eta_{i}(k)}^{i}\left(h_{i}\right)\right) \vee\left(\mathrm{v}_{j \in J} \mathcal{B}_{\eta_{j}(k)}^{j}\left(g_{j}\right)\right) .
$$

Put for $m \in I \cup J$,

$$
p_{m}=h_{m} \text {, if } m \in I \backslash(I \cap J) ; p_{m}=g_{m} \text {, if } m \in J \backslash(I \cap J) ; p_{m}=h_{m} \cup g_{m}, m \in(I \cap J) \text {. }
$$

Since for each $m \in I \cap J$;

$$
\left\langle\mathcal{B}^{m}\right\rangle_{k}\left(h_{m} \cup g_{m}\right) \leq \mathcal{B}_{k}^{m}\left(h_{m}\right) \vee \mathcal{B}_{k}^{m}\left(g_{m}\right)
$$

Then we have

$$
\langle\mathcal{B}\rangle_{k}\left(f_{1} \sqcup f_{2}\right) \nsubseteq\left(\vee_{m \in(I \cup J) \backslash(I \cap J)} \mathcal{B}_{\eta_{m}(k)}\left(p_{m}\right)\right) \vee\left(\vee_{m \in I \cap J}\left\langle\mathcal{B}^{m}\right\rangle_{\eta_{m}(k)}\left(h_{m} \cup g_{m}\right)\right)
$$

From the definition of $\left\langle\mathcal{B}^{m}\right\rangle_{\eta_{m}(k)}$, there exists $q_{m} \in\left(L^{X_{m}}\right)^{E_{m}}$ with $h_{m} \sqcup g_{m} \subseteq q_{m}$ such that

$$
\langle\mathcal{B}\rangle_{k}\left(f_{1} \sqcup f_{2}\right) \nsubseteq\left(\mathrm{v}_{m \in(I \cup J) \backslash(I \cap J)} \mathcal{B}_{\eta_{m}(k)}^{m}\left(p_{m}\right)\right) \vee\left(\mathrm{v}_{m \in I \cap J} \mathcal{B}_{\eta_{m}(k)}^{m}\left(q_{m}\right)\right) \text {. }
$$

On the other hand,

$$
\begin{gathered}
f_{1} \sqcup f_{2}=\left(\sqcup_{i \in I}\left(\varphi_{\psi}\right)_{i}^{-1}\left(h_{i}\right)\right) \sqcup\left(\sqcup_{j \in J}\left(\varphi_{\psi}\right)_{j}^{-1}\left(g_{j}\right)\right) \\
f_{1} \sqcup f_{2} \sqsubseteq\left(\sqcup_{m \in(I \cup J) \backslash(I \cap J)}\left(\varphi_{\psi}\right)_{m}^{-1}\left(p_{m}\right)\right) \sqcup\left(\sqcup_{m \in I \cap J}\left(\varphi_{\psi}\right)_{m}^{-1}\left(q_{m}\right)\right),
\end{gathered}
$$

and since $I \sqcap J$ is finite,

$$
\langle\mathcal{B}\rangle_{k}\left(f_{1} \sqcup f_{2}\right) \leq\left(\mathrm{v}_{m \in(I \cup J) \backslash(I \cap J)} \mathcal{B}_{\eta_{m}(k)}^{m}\left(p_{m}\right)\right) \vee\left(\mathrm{v}_{m \in I \cap J} \mathcal{B}_{\eta_{m}(k)}^{m}\left(q_{m}\right)\right) .
$$


It is a contradiction. Hence $\mathcal{B}$ is an $(L, M)$-fuzzy $(E, K)$-soft grill base on $X$.

(2) Necessity of the composition condition is clear, since the composition of grill maps is a grill map. Conversely, for each finite index set $I$ with $g \sqsubseteq \sqcup_{i \in I}\left(\varphi_{\psi}\right)_{i}^{-1}\left(h_{i}\right)$, since for each $i \in I$, $\left(\varphi_{\psi, \eta}\right)_{i} \circ \varphi_{\psi, \eta}:\left(Y, \mathcal{G}^{\prime}\right) \rightarrow\left(X_{i},\left\langle\mathcal{B}^{i}\right\rangle\right)$ is a grill map, where $\left(Y, \mathcal{G}^{\prime}\right)$ is an $(L, M)$-fuzzy $\left(E^{*}, K^{*}\right)$-soft grill, we have

$$
\left\langle\mathcal{B}^{i}\right\rangle_{\left(\eta \circ \eta^{*}\right)\left(k^{*}\right)}\left(h_{i}\right) \geq \mathcal{G}_{k^{*}}^{\prime}\left(\left(\left(\varphi_{\psi}\right)_{i} \circ \varphi_{\psi}\right)^{-1}\left(h_{i}\right)\right)
$$

For each $k^{*} \in K^{*}$. Since $\varphi_{\psi}^{-1}(g) \sqsubseteq \varphi_{\psi}^{-1}\left(\sqcup_{i \in I}\left(\varphi_{\psi}\right)_{i}^{-1}\left(h_{i}\right)\right)$, we have

$$
\mathcal{G}_{k^{*}}^{\prime}\left(\varphi_{\psi}^{-1}(g)\right) \leq \mathrm{v}_{i \in I} \mathcal{G}_{k^{*}}^{\prime}\left(\varphi_{\psi}^{-1}\left(\left(\varphi_{\psi}\right)_{i}^{-1}\left(h_{i}\right)\right)\right) \leq \mathrm{v}_{i \in I}\left\langle\mathcal{B}^{i}\right\rangle_{\eta_{i}\left(k^{*}\right)}\left(h_{i}\right) \leq \mathrm{v}_{i \in I} \mathcal{B}_{\eta_{i}\left(k^{*}\right)}^{i}\left(h_{i}\right)
$$

By the definition of $\langle\mathcal{B}\rangle$, we have

$\langle\mathcal{B}\rangle_{\eta\left(k^{*}\right)}(g) \geq \mathcal{G}_{k^{*}}^{\prime}\left(\varphi_{\psi}^{-1}(g)\right)$, for each $k^{*} \in K^{*}$.

(3) Put $\mathcal{G}=\cup_{i \in \Gamma}\left(\left(\left(\varphi_{\psi, \eta}\right)_{i}\right)_{1}^{\in}\right)\left(\left\langle\mathcal{B}^{i}\right\rangle\right)$, by applying (1) to both $\langle\mathcal{B}\rangle$ and $\langle\mathcal{G}\rangle$, we get the related equality.

Corollary 4.1 Let $\left\{\mathcal{B}^{i}\right\}_{i \in \Gamma}$ be a family of $(L, M)$-fuzzy $(E, K)$-soft grill bases on $X$ satisfying the following condition:

(C) For every finite subset $I$ of $\Gamma$, if $\sqcup_{i \in I} f_{i}=\tilde{E}_{X}$, then $\vee_{i \in I} \mathcal{B}_{k}^{i}\left(f_{i}\right)=1_{M}$, for all $k \in K$.

We define a map $\cup_{i \in \Gamma} \mathcal{B}^{i}: K \rightarrow M^{\left(L^{X}\right)^{E}}$ as follows:

$$
\cup_{i \in \Gamma} \mathcal{B}_{k}^{i}(g)=\Lambda\left\{\vee_{i \in I} \mathcal{B}_{k}^{i}\left(g_{i}\right) \mid g=\sqcup_{i \in I} g_{i}\right\},
$$

where the infimum is taken for every finite subset $I$ of $\Gamma$ such that $g=\sqcup_{i \in I} g_{i}$. Then $\cup_{i \in \Gamma} \mathcal{B}^{i}$ is an $(L, M)$-fuzzy $(E, K)$-soft grill base on $X$ and $\left\langle\cup_{i \in \Gamma} \mathcal{B}^{i}\right\rangle$ is the coarsest $(L, M)$-fuzzy $(E, K)$-soft grill finer than $\left\langle\mathcal{B}^{i}\right\rangle$ for each $i \in \Gamma$.

Corollary 4.2 Let $X=\Pi_{i \in \Gamma} X_{i}$ be a product set, $p_{i}: X \rightarrow X_{i}, q_{i}: E \rightarrow E_{i}$ and $r_{i}: K \rightarrow K_{i}$ be projection functions, for all $i \in \Gamma$. Let $\left\{\mathcal{B}^{i}\right\}_{i \in \Gamma}$ be a family of $(L, M)$-fuzzy $\left(E_{i}, K_{i}\right)$-soft grill bases on $X_{i}$ satisfying the following condition:

(C) For every finite subset $I$ of $\Gamma$, if $\sqcup_{i \in I}\left(p_{q}\right)_{i}^{-1}\left(h_{i}\right)=\tilde{E}_{X}$, then $\vee_{i \in I} \mathcal{B}_{k^{*}}^{i}\left(h_{i}\right)=1_{M}$, for all $k^{*} \in K_{i}$.

We define a map $\mathcal{B}$ from $K$ to $M^{\left(L^{X}\right)^{E}}$ as follows:

$$
\mathcal{B}_{k}(g)=\wedge\left\{\mathrm{v}_{i \in I} \mathcal{B}_{r_{i}(k)}^{i}\left(g_{i}\right) \mid g=\sqcup_{i \in I}\left(p_{q}\right)_{i}^{-1}\left(g_{i}\right)\right\}
$$

where the infimum is taken for every finite subset $I$ of $\Gamma$ such that $g=\sqcup_{i \in I}\left(p_{q}\right)_{i}^{-1}\left(g_{i}\right)$. Then the followings are valid:

(1) $\mathcal{B}$ is an $(L, M)$-fuzzy $(E, K)$-soft grill base on $X$ and $\langle\mathcal{B}\rangle$ is the coarsest $(L, M)$-fuzzy $(E, K)$-soft grill on $X$ for which $\left(p_{q, r}\right)_{i}:(X,\langle\mathcal{B}\rangle) \rightarrow\left(X_{i},\left\langle\mathcal{B}^{i}\right\rangle\right)$ is a grill map.

(2) $\varphi_{\psi, \eta}:\left(Y, \mathcal{G}^{\prime}\right) \rightarrow(X,\langle\mathcal{B}\rangle)$ is a grill map if and only if for each $i \in \Gamma,\left(p_{q, r}\right)_{i} \circ \varphi_{\psi, \eta}:\left(Y, \mathcal{G}^{\prime}\right) \rightarrow$ $\left(X_{i},\left\langle\mathcal{B}^{i}\right\rangle\right)$ is a grill map. 
In Corollary 4.2, the structure $\mathcal{B}$ is called the product $(L, M)$-fuzzy $(E, K)$-soft grill on $X$.

Proposition 4.1 Let $\varphi: X_{1} \rightarrow X_{2}$ and $\psi: E_{1} \rightarrow E_{2}$ be bijective functions and let $\mathcal{B}$ be an $(L, M)$-fuzzy ( $\left.E_{1}, K_{1}\right)$-soft grill base on $X_{1}$. Then we have the following properties:

(1) $\left(\varphi_{\psi, \eta}\right)_{2}^{\Rightarrow}(\mathcal{B})$ is an $(L, M)$-fuzzy $\left(E_{2}, K_{2}\right)$-soft grill base on $X_{2}$ and $\left\langle\left(\varphi_{\psi, \eta}\right)_{2}^{\Rightarrow}(\mathcal{B})\right\rangle$ is the coarsest $(L, M)$-fuzzy $\left(E_{2}, K_{2}\right)$-soft grill for which $\varphi_{\psi, \eta}:\left(X_{1},\langle\mathcal{B}\rangle\right) \rightarrow\left(X_{2},\left\langle\left(\varphi_{\psi, \eta}\right)_{2}(\mathcal{B})\right\rangle\right)$ is a grill preserving map.

(2) $\left(\varphi_{\psi, \eta}\right)_{1}^{\in}\left(\left(\varphi_{\psi, \eta}\right)_{2}^{\Rightarrow}(\mathcal{B})\right) \quad$ is $\quad$ an $(L, M)$-fuzzy $\left(E_{1}, K_{1}\right)$-soft grill base on $X_{1} \quad$ with $\left(\varphi_{\psi, \eta}\right)_{1}^{\Leftarrow}\left(\left(\varphi_{\psi, \eta}\right)_{2}^{\Rightarrow}(\mathcal{B})\right)=\mathcal{B}$.

Proof. (1) (GB1) is obvious.

(GB2) Suppose there exist $h_{1}, h_{2} \in\left(L^{X_{2}}\right)^{E_{2}}$ and $k^{*} \in K_{2}$ such that

$$
\left\langle\left(\varphi_{\psi, \eta}\right)_{2}^{\Rightarrow}(\mathcal{B})\right\rangle_{k^{*}}\left(h_{1} \sqcup h_{2}\right) \not\left(\varphi_{\psi, \eta}\right)_{2}^{\Rightarrow}(\mathcal{B})_{k^{*}}\left(h_{1}\right) \vee\left(\varphi_{\psi, \eta}\right)_{2}^{\Rightarrow}(\mathcal{B})_{k^{*}}\left(h_{2}\right) .
$$

By the definition of $\left(\varphi_{\psi, \eta}\right)_{2}^{\Rightarrow}(\mathcal{B})$ for each $k \in K_{1}$ with $k^{*}=\eta(k)$ we have

$$
\left\langle\left(\varphi_{\psi, \eta}\right)_{2}^{\Rightarrow}(\mathcal{B})\right\rangle_{k^{*}}\left(h_{1} \sqcup h_{2}\right) \not \mathcal{B}_{k}\left(\varphi_{\psi}^{-1}\left(h_{1}\right)\right) \vee \mathcal{B}_{k}\left(\varphi_{\psi}^{-1}\left(h_{2}\right)\right) .
$$

Since $\mathcal{B}$ is an $(L, M)$-fuzzy $\left(E_{2}, K_{2}\right)$-soft grill base, i.e.,

$$
\langle\mathcal{B}\rangle_{k}\left(\varphi_{\psi}^{-1}\left(h_{1} \sqcup h_{2}\right)\right) \leq \mathcal{B}_{k}\left(\varphi_{\psi}^{-1}\left(h_{1}\right)\right) \vee \mathcal{B}_{k}\left(\varphi_{\psi}^{-1}\left(h_{2}\right)\right) .
$$

Hence, we get

$$
\left\langle\left(\varphi_{\psi, \eta}\right)_{2}^{\Rightarrow}(\mathcal{B})\right\rangle_{k^{*}}\left(h_{1} \sqcup h_{2}\right) \nsubseteq \mathcal{B}_{k}\left(\varphi_{\psi}^{-1}\left(h_{1}\right)\right) \vee \mathcal{B}_{k}\left(\varphi_{\psi}^{-1}\left(h_{2}\right)\right) .
$$

By the definition of $\langle\mathcal{B}\rangle$, there exists $g \in\left(L^{X_{1}}\right)^{E_{1}}$ with $h_{1} \sqcup h_{2} \sqsubseteq g$ such that

$$
\left\langle\left(\varphi_{\psi, \eta}\right)_{2}^{\Rightarrow}(\mathcal{B})\right\rangle_{\eta(k)}\left(h_{1} \sqcup h_{2}\right) \not \mathcal{B}_{k}(g) \text {. }
$$

Since $h_{1} \sqcup h_{2}=\varphi_{\psi}\left(\varphi_{\psi}^{-1}\left(h_{1} \sqcup h_{2}\right)\right) \sqsubseteq \varphi_{\psi}(g)$ and $\varphi_{\psi}$ is injective,

$$
\left\langle\left(\varphi_{\psi, \eta}\right)_{2}^{\Rightarrow}(\mathcal{B})\right\rangle_{\eta(k)}\left(h_{1} \sqcup h_{2}\right) \leq\left\langle\left(\varphi_{\psi, \eta}\right)_{2}^{\Rightarrow}(\mathcal{B})\right\rangle_{\eta(k)}\left(\varphi_{\psi}(g)\right)=\mathcal{B}_{k}\left(\varphi_{\psi}^{-1}\left(\varphi_{\psi}(g)\right)\right) \text {. }
$$

Hence, we have

$$
\left\langle\left(\varphi_{\psi, \eta}\right)_{2}^{\Rightarrow}(\mathcal{B})\right\rangle_{\eta(k)}\left(h_{1} \sqcup h_{2}\right) \leq \mathcal{B}_{k}(g) .
$$

It is a contradiction. Hence $\left(\varphi_{\psi, \eta}\right)_{2}^{\Rightarrow}(\mathcal{B})$ is an $(L, M)$-fuzzy $\left(E_{2}, K_{2}\right)$-soft grill base on $X_{2}$. Other cases are similarly proved as in Theorem 4.1 (1).

(2) From the condition of Theorem $4.1(1)$, we have $\varphi_{\psi}^{-1}(h)=\Phi$ implies

$$
\left(\varphi_{\psi, \eta}\right)_{2}^{\Rightarrow}(\mathcal{B})_{k^{*}}(h)=\mathrm{v}_{k^{*}=\eta(k)} \mathcal{B}_{k}\left(\varphi_{\psi}^{-1}(h)\right)=0_{M}
$$

Thus, $\left(\varphi_{\psi, \eta}\right)_{1}^{\Leftarrow}\left(\left(\varphi_{\psi, \eta}\right)_{2}^{\Rightarrow}(\mathcal{B})\right)$ is an $(L, M)$-fuzzy $\left(E_{1}, K_{1}\right)$-soft grill base on $X_{1}$. By an easy assumption the equality $\left(\varphi_{\psi, \eta}\right)_{1}^{\models}\left(\left(\varphi_{\psi, \eta}\right)_{2}^{\Rightarrow}(\mathcal{B})\right)=\mathcal{B}$ is obtained.

Theorem 4.4 Let $\varphi: X_{i} \rightarrow X$ and $\psi: E_{i} \rightarrow E$ be injective functions for all $i \in \Gamma$. Let $\left\{\mathcal{B}^{i}\right\}_{i \in \Gamma}$ be a family of $(L, M)$-fuzzy $\left(E_{i}, K_{i}\right)$-soft grill bases on $X_{i}$ satisfying the following condition: 
(C) For every finite subset $I$ of $\Gamma$, if $\sqcup_{i \in I} g_{i}=\tilde{E}$, then $\vee_{i \in I}\left(\left(\varphi_{\psi, \eta}\right)_{i}\right)_{2}\left(\mathcal{B}^{i}\right)_{k^{*}}\left(g_{i}\right)=1_{M}$, for each $k^{*} \in K_{i}$.

Let $\mathcal{B}: K \rightarrow M^{\left(L^{X}\right)^{E}}$ be a mapping defined as follows.

$$
\mathcal{B}_{k}(g)=\Lambda\left\{\mathrm{v}_{i \in I}\left(\left(\varphi_{\psi}\right)_{i}\right)_{2}^{\Rightarrow}\left(\mathcal{B}^{i}\right)_{k}\left(g_{i}\right) \mid g=\sqcup_{i \in I} g_{i}\right\}
$$

where the infimum is taken for every finite subset $I$ of $\Gamma$. Then the following properties are satisfied.

(1) $\mathcal{B}$ is an $(L, M)$-fuzzy $(E, K)$-soft grill base on $X$ and $\langle\mathcal{B}\rangle$ is the coarsest $(L, M)$-fuzzy $(E, K)$-soft grill for which $\left(\varphi_{\psi, \eta}\right)_{i}:\left(X_{i},\left\langle\mathcal{B}^{i}\right\rangle\right) \rightarrow(X,\langle\mathcal{B}\rangle)$ is a grill preserving map.

(2) $\varphi_{\psi, \eta}:(X,\langle\mathcal{B}\rangle) \rightarrow(Y, \mathcal{G})$ is a grill preserving map iff $\varphi_{\psi, \eta} \circ\left(\varphi_{\psi, \eta}\right)_{i}:\left(X_{i},\left\langle\mathcal{B}^{i}\right\rangle\right) \rightarrow(Y, \mathcal{G})$ is a grill preserving map for each $i \in \Gamma$.

Proof. (1) From Corollary 4.2 and Proposition $4.1, \mathcal{B}$ is an $(L, M)$-fuzzy $(E, K)$-soft grill base on $X$. Since $\left(\varphi_{\psi}\right)_{i}$ is injective for all $i \in \Gamma$,

$$
\mathcal{B}_{\eta_{i}\left(k_{i}\right)}\left(\left(\varphi_{\psi}\right)_{i}\left(f_{i}\right)\right) \leq\left(\left(\varphi_{\psi}\right)_{i}\right)_{2}^{\Rightarrow}\left(\mathcal{B}^{i}\right)_{\eta_{i}\left(k_{i}\right)}\left(\left(\varphi_{\psi}\right)_{i}\left(f_{i}\right)\right)=\mathcal{B}_{k_{i}}^{i}\left(\left(\varphi_{\psi}\right)_{i}^{-1}\left(\left(\varphi_{\psi}\right)_{i}\left(f_{i}\right)\right)\right)=\mathcal{B}_{k_{i}}^{i}\left(f_{i}\right) .
$$

Hence $\left(\varphi_{\psi}\right)_{i}$ is a grill preserving map for each $i \in \Gamma$. Other cases are similarly proved as in Theorem $4.3(1)$.

(2) It is similarly proved as in Theorem 4.3 (2).

Theorem 4.5 Let $\varphi: X_{1} \rightarrow X_{2}, \psi: E_{1} \rightarrow E_{2}$ be surjective functions and $\mathcal{B}$ be an $(L, M)$-fuzzy $\left(E_{1}, K_{1}\right)$-soft grill base on $X_{1}$. Then we have the following properties:

(1) $\left(\varphi_{\psi, \eta}\right)_{1}^{\Rightarrow}(\mathcal{B})$ is an $(L, M)$-fuzzy $\left(E_{2}, K_{2}\right)$-soft grill base on $X_{2}$.

(2) $\left\langle\left(\varphi_{\psi, \eta}\right)_{1}^{\Rightarrow}(\mathcal{B})\right\rangle$ is the coarsest $(L, M)$-fuzzy $\left(E_{2}, K_{2}\right)$-soft gril on $X_{2}$ for which $\varphi_{\psi, \eta}:\left(X_{1},\langle\mathcal{B}\rangle\right) \rightarrow$ $\left(X_{2},\left\langle\left(\varphi_{\psi, \eta}\right)_{1}^{\Rightarrow}(\mathcal{B})\right\rangle\right)$.

(3) If $\varphi_{\psi}$ is bijective and $\mathcal{B}$ is an $(L, M)$-fuzzy $\left(E_{1}, K_{1}\right)$-soft grill on $X_{1}$, then $\left\langle\left(\varphi_{\psi, \eta}\right)_{1}^{\Rightarrow}(\mathcal{B})\right\rangle=\left(\varphi_{\psi, \eta}\right)_{2}^{\Rightarrow}(\mathcal{B})$.

Proof. (1) Similar to the proof of Theorem 4.1(1).

(2) Clear from the equality $\left\langle\left(\varphi_{\psi, \eta}\right)_{1}^{\Rightarrow}(\mathcal{B})\right\rangle_{k^{*}}\left(\tilde{E}_{X_{2}}\right)=\bigvee_{k^{*}=\eta(k)} \mathcal{B}_{k}\left(\tilde{E}_{X_{1}}\right)=1_{M}$.

(3) Let $\varphi_{\psi}$ be bijective and let $\mathcal{B}$ be an $(L, M)$-fuzzy $\left(E_{1}, K_{1}\right)$-soft grill on $X_{1}$ Since $h \sqsubseteq \varphi_{\psi}(f)$ iff $\varphi_{\psi}^{-1}(h) \subseteq f$ for which $h \in\left(L^{X_{2}}\right)^{E_{2}}$, we have

$$
\begin{aligned}
& \left\langle\left(\varphi_{\psi, \eta}\right)_{1}^{\Rightarrow}(\mathcal{B})\right\rangle_{k^{*}}(h)=\Lambda\left\{\left(\varphi_{\psi, \eta}\right)_{1}^{\Rightarrow}(\mathcal{B})_{k^{*}}(g) \mid h \sqsubseteq g\right\}=\wedge\left\{\bigvee_{k^{*}=\eta(k)} \mathcal{B}_{k}(f) \mid h \sqsubseteq g=\varphi_{\psi}(f)\right\} \\
& \quad=\Lambda\left\{\bigvee_{k^{*}=\eta(k)} \mathcal{B}_{k}(f) \mid \varphi_{\psi}^{-1}(h) \sqsubseteq f\right\}=\bigvee_{k^{*}=\eta(k)} \mathcal{B}_{k}\left(\varphi_{\psi}^{-1}(h)\right)= \\
& \left(\varphi_{\psi, \eta}\right)_{2}^{\Rightarrow}(\mathcal{B})_{k^{*}}(h) .
\end{aligned}
$$


Theorem 4.6 Let $\varphi_{i}: X_{i} \rightarrow X, \psi_{i}: E_{i} \rightarrow E$ and $\eta_{i}: K_{i} \rightarrow K$ be functions for each $i \in \Gamma$. Let $\left\{\mathcal{B}^{i}\right\}_{i \in \Gamma}$ be a family of $(L, M)$-fuzzy $\left(E_{i}, K_{i}\right)$-soft grill bases on $X_{i}$ satisfying the following condition:

(C) For every finite subset $I$ of $\Gamma$, if $\sqcup_{i \in I}\left(\varphi_{\psi}\right)_{i}\left(g_{i}\right)=\tilde{E}_{X}$, then $\bigvee_{i \in I} \mathcal{B}^{i}{ }^{*}\left(g_{i}\right)=1_{M}$ for each $k^{*} \in K_{i}$.

We define a mapping $\biguplus_{i \in I}\left(\left(\varphi_{\psi, \eta}\right)_{i}\right)_{1}^{\Rightarrow}\left(\mathcal{B}^{i}\right): K \rightarrow M^{\left(L^{X}\right)^{E}}$ as

$$
\left(\biguplus_{i \in I}\left(\left(\varphi_{\psi, \eta}\right)_{i}\right)_{1}^{\Rightarrow}\left(\mathcal{B}^{i}\right)\right)_{k}(h)=\Lambda\left\{\bigvee_{i \in I} \bigvee_{k=\eta_{i}\left(k^{*}\right)} \mathcal{B}_{k^{*}}^{i}\left(g_{i}\right) \mid h=\sqcup_{i \in I}\left(\varphi_{\psi}\right)_{i}\left(g_{i}\right)\right\},
$$

where the infimum is taken for every finite subset $I$ of $\Gamma$. Then the following properties are satisfied:

(1) If $\varphi_{j}$ and $\psi_{j}$ are surjective for some $j \in \Gamma$, then $\mathcal{B}=\biguplus_{i \in I}\left(\left(\varphi_{\psi, \eta}\right)_{i}\right)_{1}^{\Rightarrow}\left(\mathcal{B}^{i}\right)$ is an $(L, M)$-fuzzy $(E, K)$-soft grill base on $X$ and $\langle\mathcal{B}\rangle$ is the coarsest $(L, M)$-fuzzy $(E, K)$-soft grill for which $\left(\varphi_{\psi, \eta}\right)_{i}:\left(X_{i},\left\langle\mathcal{B}^{i}\right\rangle\right) \rightarrow(X,\langle\mathcal{B}\rangle)$ is a grill preserving map.

(2) A map $\varphi_{\psi, \eta}:(X,\langle\mathcal{B}\rangle) \rightarrow(Y, \mathcal{G})$ is a grill preserving map if and only if $\varphi_{\psi, \eta}$ 。 $\left(\varphi_{\psi, \eta}\right)_{i}:\left(X_{i},\left\langle\mathcal{B}^{i}\right\rangle\right) \rightarrow(Y, \mathcal{G})$ is a grill preserving map for each $i \in \Gamma$.

(3) If $\varphi_{j}$ and $\psi_{j}$ are surjective for all $j \in \Gamma$, then $\left\langle\biguplus_{i \in I}\left(\left(\varphi_{\psi, \eta}\right)_{i}\right)_{1}^{\Rightarrow}\left(\mathcal{B}^{i}\right)\right\rangle=\left\langle\bigcup_{i \in I}\left(\left(\varphi_{\psi, \eta}\right)_{i}\right)_{1}{ }_{1}\left(\mathcal{B}^{i}\right)\right\rangle$.

Proof. (1) (GB1) Since $\varphi_{j}$ and $\psi_{j}$ are surjective for some $j \in \Gamma$ and $(\mathrm{C}), \mathcal{B}_{k}\left(\widetilde{E}_{X}\right)=1_{M}$ and $\mathcal{B}_{k}\left(\Phi_{X}\right)=0_{M}$. The other cases are similar to the proof of Theorem 4.3(1).

(2) Similarly proved as in Theorem 4.3(2).

(3) We show that the following condition (C1) and (C) are equivalent:

(C1) For every finite subset $I$ of $\Gamma$, if $\sqcup_{i \in I} h_{i}=\tilde{E}_{X}$, then $\bigvee_{i \in I}\left(\left(\varphi_{\psi, \eta}\right)_{i}\right)_{1}^{\Rightarrow}\left(\mathcal{B}^{i}\right)_{k}\left(h_{i}\right)=1_{M}$ for each $k \in K$.

$(\mathrm{C} 1) \Rightarrow(\mathrm{C})$ : For every finite subset $I$ of $\Gamma$, if $\sqcup_{i \in I}\left(\varphi_{\psi}\right)_{i}\left(g_{i}\right)=\tilde{E}_{X}$, by $(\mathrm{C} 1)$,

$$
1_{M}=\bigvee_{i \in I}\left(\left(\varphi_{\psi, \eta}\right)_{i}\right)_{1}^{\Rightarrow}\left(\mathcal{B}^{i}\right)_{k}\left(\left(\varphi_{\psi}\right)_{i}\left(g_{i}\right)\right) \leq \bigvee_{i \in I} \bigvee_{k=\eta_{i}\left(k^{*}\right)} \mathcal{B}_{k^{*}}^{i}\left(g_{i}\right)
$$

(C) $\Rightarrow(\mathrm{C} 1)$ : If $\vee_{i \in I}\left(\left(\varphi_{\psi, \eta}\right)_{i}\right)_{1}^{\Rightarrow}\left(\mathcal{B}^{i}\right)_{k}\left(h_{i}\right) \neq 1_{M}$, for each $i \in I$, there exists $g_{i} \in\left(L^{X_{i}}\right)^{E_{i}}$ with $h_{i}=$ $\left(\varphi_{\psi}\right)_{i}\left(g_{i}\right)$ such that

$$
\bigvee_{i \in I}\left(\left(\varphi_{\psi, \eta}\right)_{i}\right)_{1}^{\Rightarrow}\left(\mathcal{B}^{i}\right)_{k}\left(h_{i}\right) \leq \bigvee_{i \in I} \bigvee_{k=\eta_{i}\left(k^{*}\right)} \mathcal{B}_{k^{*}}^{i}\left(g_{i}\right) \neq 1_{M}
$$

$\operatorname{By}(\mathrm{C}), \sqcup_{i \in I}\left(\varphi_{\psi}\right)_{i}\left(g_{i}\right)=\sqcup_{i \in I} h_{i} \neq \tilde{E}_{X}$.

For each finite index set $I$ with $\left\{g_{i} \mid h \sqsubseteq \sqcup_{i \in I}\left(\varphi_{\psi}\right)_{i}\left(g_{i}\right)\right\}$, by the definition of $\left\langle\bigcup_{i \in I}\left(\left(\varphi_{\psi, \eta}\right)_{i}\right)_{1}^{\Rightarrow}\left(\mathcal{B}^{i}\right)\right\rangle$, we have

$$
\left\langle\cup_{i \in I}\left(\left(\varphi_{\psi, \eta}\right)_{i}\right)_{1}^{\Rightarrow}\left(\mathcal{B}^{i}\right)\right\rangle_{k}(h) \leq \bigwedge_{i \in \Gamma}\left(\left(\varphi_{\psi, \eta}\right)_{i}\right)_{1}^{\Rightarrow}\left(\mathcal{B}^{i}\right)_{k}\left(\sqcup_{i \in I}\left(\varphi_{\psi}\right)_{i}\left(g_{i}\right)\right) \leq \bigvee_{i \in I} \bigvee_{k=\eta_{i}\left(k^{*}\right)} \mathcal{B}_{k^{*}}^{i}\left(g_{i}\right)
$$


Hence $\left\langle\biguplus_{i \in I}\left(\left(\varphi_{\psi, \eta}\right)_{i}\right)_{1}^{\Rightarrow}\left(\mathcal{B}^{i}\right)\right\rangle \geq\left\langle\bigcup_{i \in I}\left(\left(\varphi_{\psi, \eta}\right)_{i}\right)_{1}^{\Rightarrow}\left(\mathcal{B}^{i}\right)\right\rangle$.

For each finite index set $J$ with $\left\{h_{j} \mid p \sqsubseteq \sqcup_{j \in J} h_{j}\right\}$, since $\varphi_{j}$ and $\psi_{j}$ are surjective for each $j \in J$, there exists $p_{j} \in\left(L^{X_{j}}\right)^{E_{j}}$ with $\left(\varphi_{\psi}\right)_{j}\left(p_{j}\right)=h_{j}$ such that $p \sqsubseteq \sqcup_{j \in J} h_{j}=\sqcup_{j i n J}\left(\varphi_{\psi}\right)_{j}\left(p_{j}\right)$. Thus

$$
\left\langle\biguplus_{i \in I}\left(\left(\varphi_{\psi, \eta}\right)_{i}\right)_{1} \Rightarrow\left(\mathcal{B}^{i}\right)\right\rangle_{k}(p) \leq \bigvee_{j \in J}\left(\left(\varphi_{\psi, \eta}\right)_{j}\right)_{1}^{\Rightarrow}\left(\mathcal{B}^{j}\right)_{k}\left(h_{j}\right) \leq \bigvee_{j \in J} \bigvee_{k=\eta_{i}\left(k^{*}\right)} \mathcal{B}_{k^{*}}^{j}\left(p_{j}\right)
$$

Hence $\left\langle\biguplus_{i \in I}\left(\left(\varphi_{\psi, \eta}\right)_{i}\right)_{1}^{\Rightarrow}\left(\mathcal{B}^{i}\right)\right\rangle \leq\left\langle\bigcup_{i \in I}\left(\left(\varphi_{\psi, \eta}\right)_{i}\right)_{1}^{\Rightarrow}\left(\mathcal{B}^{i}\right)\right\rangle$.

\section{Conclusion}

In general topology (also in fuzzy topology), the concept of grill has been observed as an excellent tool for studying different topological structures. So, we found it reasonable to extend the notions of grill and grill base, which were introduced by Abbas [1], to the fuzzy soft case. In addition, we described the second order image and preimage operators of fuzzy soft grill base and discussed some of their structural characteristics.

\section{References}

[1] S. E. Abbas, "Images and Preimages of $(L, M)$-grillbases", Hacettepe J. of Maths. and Statistics, 40(5) (2011) 737-747.

[2] K. K. Azad, "Fuzzy grills and a characterization of fuzzy proximity", J. Math. Anal. Appl. 79 (1981) 13-17.

[3] B. Ahmad, A. Kharal, "On fuzzy soft sets, Advances in Fuzzy Systems", Vol. (2009), Article ID 586507.

[4] H. Aktaş, N. Çağman, "Soft sets and soft groups", Information Sciences 177 (13) (2007) 27262735.

[5] R. Ameri, H. Hedayati, E. Ghasemian, "Filteristic soft BCK-algebras", Journal of Mathematics and Computer Science 2(1) (2011) 81-87.

[6] A. Aygünoğlu, H. Aygün, "Introduction to fuzzy soft groups", Computers and Mathematics with Applications 58 (2009) 1279-1286.

[7] A. Aygünoğlu, V. Çetkin, H. Aygün, "An introduction to fuzzy soft topological spaces", Hacettepe Journal of Mathematics and Statistics, 43(2) (2014) 197-208.

[8] A. Bhattacharyya, M. N. Mukherjee, S. P. Sinha, "Concerning fuzzy grills: Some applications", Hacettepe J. of Math. and Statistics, 34 (2005) 91-100.

[9] K. C. Chattopadhyay, U. K. Mukherjee, S. K. Samanta, "Fuzzy proximity structures and fuzzy grills", Fuzzy Sets and Systems, 79 (1996) 383-393.

[10] G. Choquet, "Sur les notions de filter et de grills", C. R. Acad. Sci. Paris 224 (1947) 171-173.

[11] Y. B. Jun, "Soft BCK/BCI algebras", Computers and Mathematics with Applications 56 (5) (2008) 1408-1413.

[12] A. Kandil, O. A. E. Tantawy, S. A. El-Sheikh, A. M. Abd El-latif, "Fuzzy semi open soft sets related properties in fuzzy soft topological spaces", Journal of Mathematics and Computer Science, 13(2) (2014) 94-114.

[13] A. Kharal, B. Ahmad, "Mappings on Fuzzy Soft Classes, Advances in Fuzzy Systems", Vol. (2009), Article ID 407890. 
[14] P. K. Maji, R. Biswas, A.R. Roy, “Fuzzy soft sets, Journal of fuzzy Mathematics”, 9(3) (2001) 589-602.

[15] P. K. Maji, A. R. Roy, R. Biswas, "An application of soft sets in a decision making problem", Computers and Mathematics with Applications 44 (8-9) (2002) 1077-1083.

[16] D. Molodtsov, "Soft set theory-First results", Computers and Mathematics with Appl. 37 (4/5) (1999) 19-31.

[17] D. Pei, D. Miao, "From soft sets to information systems", Granular Computing, 2005 IEEE International Conference on (2) (2005) 617-621.

[18] A. R. Roy, P. K. Maji, "A fuzzy soft set theoretic approach to decision making problems", Journal of Computational and Applied Mathematics, 203 (2007) 412-418.

[19] A. P. Sostak, “On a fuzzy topological structure”, Suppl. Rend. Circ. Matem. Palermo, Ser II 11 (1985) 89-103.

[20] P. Srivastava, R. L. Gupta, "Fuzzy proximity structures and fuzzy ultrafilters", J. Math. Anal. Appl. 94(2) (1983) 297-311. 